\title{
Evaluación de fuentes de fertilización edáfica y el efecto sobre la microbiota presente en un cultivo de cebolla cabezona (Allium cepa L.)
}

\author{
Evaluation of fertilization sources and the effect \\ on microbiota present in bulb onion \\ (Allium cepa L.) culture
}

CARLOS ALBERTO LUENGAS-GÓMEZ1,3

DIANA PAOLA MORA-CASTRO²

DORISANA GUERRERO-FLÓREZ1

Cultivo de cebolla cabezona

en Pasca, Cundinamarca.

Foto: D. Guerrero-Flórez

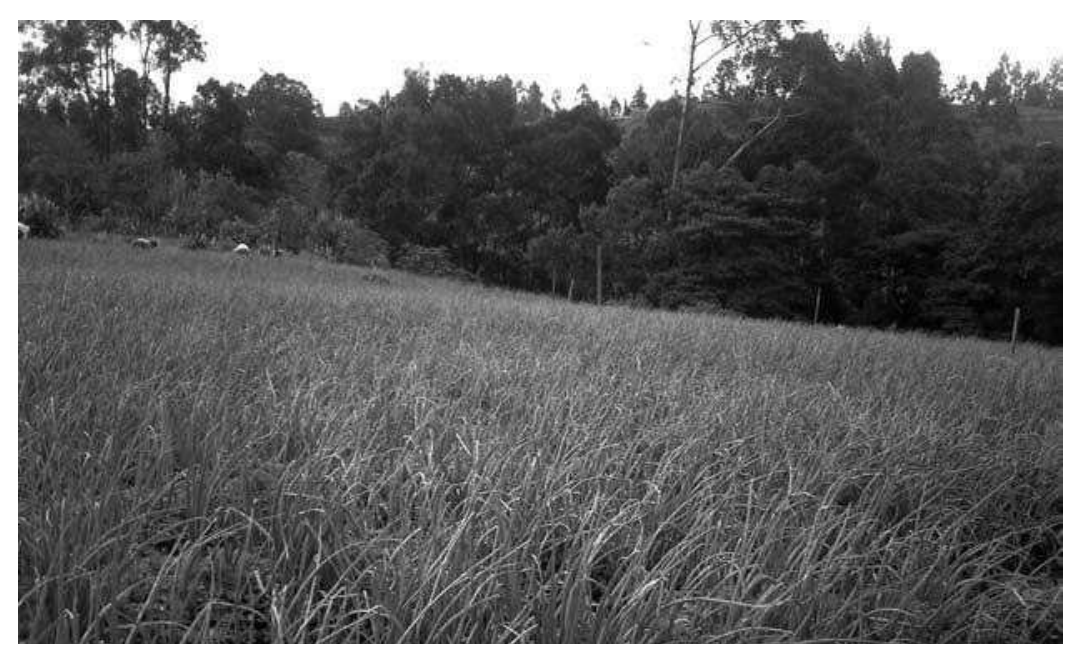

\section{RESUMEN}

En Pasca (Cundinamarca, Colombia), municipio ubicado a $2.090 \mathrm{msnm}$, temperatura y precipitaciones anuales promedio de $14^{\circ} \mathrm{C}$ y $1.800 \mathrm{~mm}$, la cebolla cabezona está distribuida ampliamente. En un lote de este municipio se estudió el híbrido Yellow Granex PRR, utilizando las siguientes fuentes y épocas de suministro para cada tratamiento; orgánico: compost, roca fosfórica, $\mathrm{KCl}, \mathrm{MgO}$ y azotobacter, incorporados en trasplante; orgánico-mineral: $\mathrm{CO}\left(\mathrm{NH}_{2}\right)_{2},\left(\mathrm{NH}_{4}\right)_{2} \mathrm{HPO}_{4}, \mathrm{MgSO}_{4}, \mathrm{KCl}, \mathrm{Ca}\left(\mathrm{NO}_{3}\right)_{2}$, Compost, adicionados en trasplante y 30 días después transplante (ddt); convencional se usaron las mismas fuentes, intercambiando el compost por micronutrientes, agregados en trasplante, 30 y $75 \mathrm{ddt}$. Se tomaron datos durante dos ciclos de cultivo consecutivos (abril 2008 a enero 2009), a cada unidad experimental se le realizaron análisis químicos de suelo y microbiológicos, al iniciar y finalizar. Se evaluaron unidades formadoras de colonia (ufc): bacterias, actinomicetos, hongos, solubilizadoras de fósforo, fijadoras de nitrógeno; se midieron variables: altura, peso fresco y seco de planta y bulbo, registros de cosecha. Los resultados mostraron respuestas favorables en rendimiento para el tratamiento orgánico en dosis media de $26,35 \mathrm{t} \mathrm{ha}^{-1}$, seguido del orgánico-mineral en dosis baja 22,65 t ha $\mathrm{a}^{-1}$ y el convencional en dosis media $19,17 \mathrm{tha}^{-1}$, se atribuye a las altas reservas minerales que posee este suelo, debido a aportes continuos de materia orgánica y cales en ciclos anteriores, justificado en todos los aná-

\footnotetext{
Universidad de Ciencias Aplicadas y Ambientales (UDCA), Bogotá (Colombia).

Universidad de Cundinamarca, Fusagasugá (Colombia).

Autor para correspondencia. cluengas@udca.edu.co
} 
lisis de suelo realizados. Se observó en el tratamiento convencional en dosis media aumento en las poblaciones evaluadas con excepción de los hongos, en el que el orgánico en dosis media reportó poblaciones superiores. En cambio, el tratamiento orgánico en dosis alta sólo aumentó para actinomicetos y fijadoras de nitrógeno, la abundancia se explica porque hubo condiciones favorables como: $\mathrm{pH}$ neutro, suelo cercano a capacidad de campo y alto contenido nutricional.

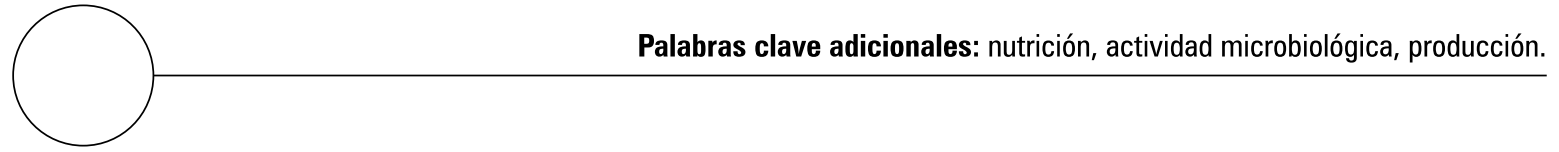

\section{ABSTRACT}

In Pasca (Cundinamarca, Colombia), municipality located at 2,090 $\mathrm{msnm}$, with annual temperature $14^{\circ} \mathrm{C}$ and precipitation average $1,800 \mathrm{~mm}$, onion bulb is widely distributed. The experimental trial was done with hybrid Yellow Granex PRR using the following sources and times for each treatment: organic that included compost, phosphoric rock, $\mathrm{KCl}, \mathrm{MgO}$ and azotobacter, built-in transplant; organo-mineral: $\mathrm{CO}\left(\mathrm{NH}_{2}\right)_{2},\left(\mathrm{NH}_{4}\right)_{2} \mathrm{HPO}_{4}$, $\mathrm{MgSO}_{4}, \mathrm{KCl}, \mathrm{Ca}\left(\mathrm{NO}_{3}\right)_{2}$, compost added at transplant and 30 days after transplanting (dat); conventional fertilizer that used the same sources, interchanging compost by micronutrients aggregated at transplanting, at 30, and 75 dat. The data were taken during two consecutive culture cycles (April 2008 to January 2009) and soil microbiological and chemical analyses were realized on each experimental unit; when initiating and finishing form units of colony (ufc) were evaluated: bacteria, actinomycota, fungi, phosphorus solubilizers, nitrogen fixers. The variables measured were: height, fresh and dry weight of plant and bulb, registries of harvest. The results showed favorable response in yield for the organic treatment in average dose $26.35 \mathrm{t}$ $\mathrm{ha}^{-1}$, followed by the organic-mineral in low dose $22.65 \mathrm{t} \mathrm{ha}^{-1}$ and conventional one in average dose $19.17 \mathrm{t}$ $\mathrm{ha}^{-1}$, it attributes to the high levels of mineral reserves that this soil has, due to the continuous contributions of organic matter and lime to it in previous cycles, justified in all realized soil analyses. An increase in the populations evaluated except that of fungi was observed in the conventional treatment in average dose, where the organic one in average dose report superior populations. However, the organic treatment in high dose only increased for actinomycota and nitrogen fixers. The abundance is explained because there were favorable conditions, such as neutral $\mathrm{pH}$, soil moisture near field capacity and high nutrient contents.

Additional keywords: nutrition, production, microbiological activity.

Fecha de recepción: 20-03-2009

Aprobado para publicación: 01-06-2009

INTRODUCCIÓN

La actividad de la agricultura que provee al suelo y planta los nutrientes faltantes, denominada fertilización, y el material aplicado lleva el nombre de fertilizante. Lo ideal es devolver al suelo todo lo extraído de él; por lo menos, el agricultor debe procurar devolver no sólo la máxima cantidad de nutrientes perdidos, sino dar alimento a los microorganismos del suelo $\mathrm{y}$, al mismo tiempo, mantener el suelo en buenas condiciones fí- sicas. La mayoría de nutrientes del suelo tienen origen en el material parental del cual se ha formado, la otra parte se incorpora por intercambio de gases, flujo de agua freática, fijación biológica de nitrógeno y mediante la fertilización.

Los microorganismos son componentes importantes del suelo, constituyen su parte viva y son responsables de la dinámica de transformación 
y desarrollo. La diversidad de microorganismos encontrados en una fracción de suelo cumple funciones determinantes en transformación de los componentes orgánicos e inorgánicos que se incorporan. La humificación de la materia orgánica es proceso netamente microbiológico. La microflora del suelo está compuesta por bacterias, actinomicetos, hongos, algas, virus y protozoarios (Abril, 2002).

El suelo utilizado en el proyecto está descrito como Humic Dystrudepts, caracterizado por un paisaje montañoso, clima frío húmedo, relieve ligeramente plano a ligeramente ondulado, pendientes $3 \%$ a $7 \%$, suelos profundos, moderadamente bien drenados, presentan reacción fuerte a fuertemente ácida, saturación de aluminio alta y fertilidad moderada a baja (Borrero, 2000).

Actualmente estas tierras del Municipio de Pasca (Cundinamarca) se encuentran dedicadas a cultivos transitorios y semipermanentes, hortalizas como la cebolla cabezona, pertenece a la familia Liliaceae, posee hojas tubulares y alargadas que dan origen al bulbo, formado por hojas modificadas (catafilas); excelente captadora de radiación solar, a su vez es ineficiente para competir con malezas. Presenta raíces cortas que salen de la parte inferior del tallo, por lo que las prácticas de riego y fertilización son muy importantes (ICA, 1989). El cultivo tiene un periodo vegetativo aproximadamente de 120 días.

A nivel nacional, la producción de este cultivo se concentra en Boyacá como el mayor productor y que registra mejores rendimientos por área $\left(23,36 \mathrm{t} \mathrm{ha}^{-1}\right)$, tercero Cundinamarca con $16,88 \mathrm{t}$ ha-1 (Melo et al., 2006).

Para las zonas de Boyacá, Cundinamarca y Nariño, el híbrido Yellow Granex tiene amplia aceptación por su tolerancia a raíz rosada y bulbos de tamaño grande forma gruesa y aplanada, sabor suave, atractivas capas exteriores.

Debido a los costos elevados de los fertilizantes sintéticos y su impacto sobre los microorganismos benéficos para las plantas, el objetivo de este trabajo fue la evaluación de diferentes fuentes y dosis de fertilización en el crecimiento y rendimiento del cultivo de cebolla para la zona de Pasca.

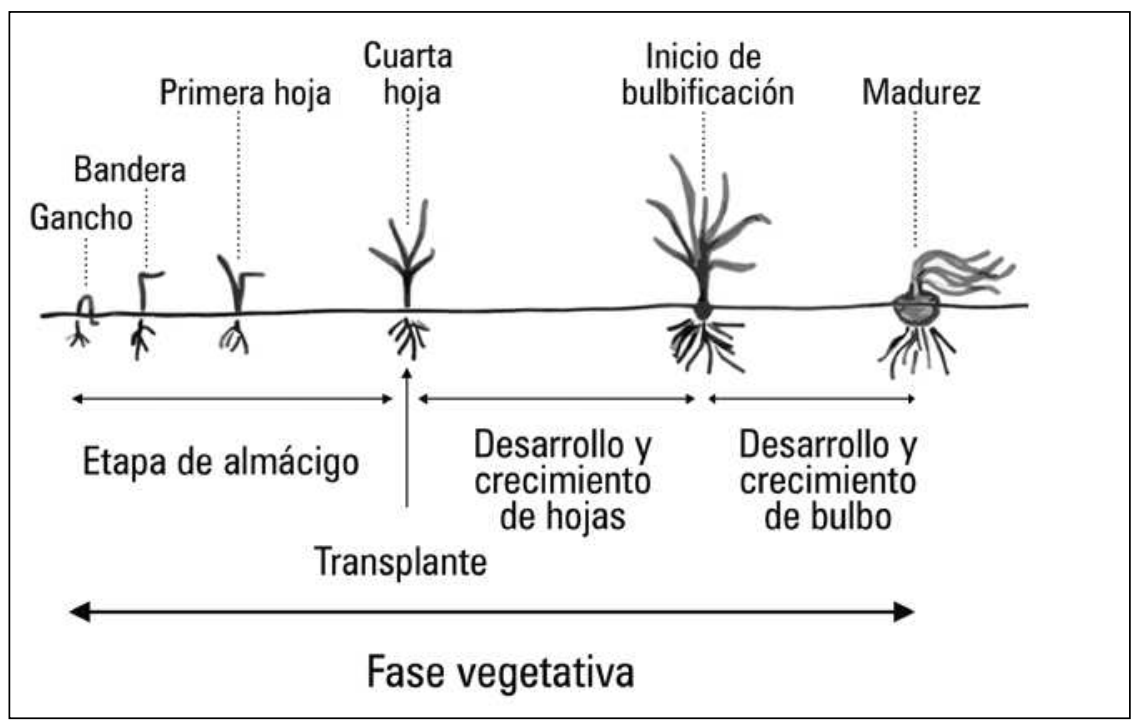

Figura 1. Estadios fenológicos de la cebolla cabezona, según Dogliotti y Galvan (2008). 


\section{MATERIALES Y MÉTODOS}

El lote donde se llevó a cabo el ensayo experimental de $2.000 \mathrm{~m}^{2}$ está localizado en el municipio de Pasca (Cundinamarca), con coordenadas geográficas de 4¹8' 45,3” N y 74¹9' 05,6” W, una altitud de $2.090 \mathrm{msnm}$, una temperatura promedio de $14,0^{\circ} \mathrm{C}$ y una precipitacion anual de $1.800 \mathrm{~mm}$.

Según el historial, hace cuatro años se cultiva el hibrido Yellow Granex (PRR) sin hacer rotación alguna, tiempo en el que se ha aplicado cal agrícola, fertilizantes químicos y altas cantidades de gallinaza para cada ciclo del cultivo, sin realizar análisis de suelo, base para efectuar un correcto plan nutricional.

El proyecto fue evaluado mediante un diseño completamente aleatorizado, utilizando las siguientes fuentes y épocas de suministro para cada tratamiento; orgánico: compost, roca fosfórica, $\mathrm{KCl}, \mathrm{MgO}$ y Azotobacter incorporados en trasplante; en la inoculación de Azotobacter, se utilizó cepa del mismo suelo, donde se realizó el adecuado aislamiento y multiplicación en laboratorios de la Universidad de Cundinamarca.

Para orgánico-mineral se usó: $\mathrm{CO}\left(\mathrm{NH}_{2}\right)_{2}$, $\left(\mathrm{NH}_{4}\right)_{2} \mathrm{HPO}_{4}, \mathrm{MgSO}_{4}, \mathrm{KCl}, \mathrm{CaNO}_{3}$, Compost, adicionados en trasplante y a los $30 \mathrm{ddt}$, el convencional contó con las mismas fuentes del anterior, intercambiando compost por menores, agregados en trasplante, $30 \mathrm{ddt}$ y $75 \mathrm{ddt}$; con dosis según extracción de la especie: 30,40 y 50 t ha ${ }^{-1}$ reportada por Bertsch (2003), para dos ciclos de cultivo.

Se obtuvieron nueve tratamientos con tres repeticiones, para un total de 27 unidades experimentales (tabla 1), evaluadas entre los meses de abril de 2008 a enero de 2009, obteniendo cosecha a mediados de agosto y finales de enero.

Se realizaron tres análisis químicos de suelos antes del trasplante, para determinar algún tipo de
Tabla 1. Tratamientos establecidos.

\begin{tabular}{|r|l|l|}
\hline No. & \multicolumn{1}{|c|}{ Tratamiento } & \multicolumn{1}{|c|}{ Abreviación } \\
\hline 1 & Orgánico alto & Org. alto \\
\hline 2 & Orgánico medio & Org. medio \\
\hline 3 & Orgánico bajo & Org. bajo \\
\hline 4 & Orgánico-mineral alto & Org. min. alto \\
\hline 5 & Orgánico-mineral medio & Org. min. medio \\
\hline 6 & Orgánico-mineral bajo & Org. min. bajo \\
\hline 7 & Convencional alto & Conv. alto \\
\hline 8 & Convencional medio & Conv. medio \\
\hline 9 & Convencional bajo & Conv. bajo \\
\hline
\end{tabular}

efecto en el suelo, posteriormente al primer y segundo ciclo de cultivo, se tomó una muestra por cada tratamiento para un número de 54 análisis. En total se efectuaron 57 muestras de suelo.

Las variables fisiológicas evaluadas se tomaron quincenalmente en cada ciclo de cultivo y algunas sólo en las cosechas, en cuanto a las primeras se tienen altura (Al), peso fresco planta (PFP), peso fresco bulbo (PFB), peso seco planta (PSP) y peso seco bulbo (PSB). En cosecha se tomaron datos de peso fresco bulbo primera (PFBP), peso fresco bulbo segunda (PFBS), peso seco bulbo primera (PSBP), peso seco bulbo segunda (PSBS) y en rendimiento primera calidad (RP) y rendimiento segunda calidad (RS).

En cuanto a variables de suelo, se tuvieron en cuenta las arrojadas por los análisis de suelos, textura (Bouyucus), pH (Potenciómetro relación 1:1), fósforo (Bray II), carbono orgánico (Walkley Black modificado), bases intercambiables, CIC (extracción acetato de amonio $1 \mathrm{~N}$ neutro), aluminio y acidez intercambiable (extracción con $\mathrm{KCl} 1 \mathrm{~N}$, titulación), densidad aparente por método del terrón parafinado.

El análisis microbiológico incluyó grupos de hongos, bacterias, actinomicetos, solubilizadoras de fósforo y fijadoras de nitrógeno cuantificando poblaciones unidades formadoras de colonia (ufc). 
La cuantificación de las ufc realizada mediante la técnica de conteo microbiano, para conocer el número de microorganismos viables en el suelo, realizando dilución y vaciado en placa de agar (Ferrera-Cerrato et al., 2007).

El análisis estadístico se realizó mediante el programa estadístico SSPS 15.

\section{RESULTADOS Y DISCUSIÓN}

\section{Parámetros de crecimiento y producción}

Según la tabla 2, no se obtuvieron respuestas a las aplicaciones de fertilizantes en las variables peso seco planta y rendimiento segunda, las demás variables analizadas presentaron respuesta significativa y en ocasiones altamente significativa a diferentes dosis y tipos de mezclas.

Se observó el caso del tratamiento Org. alto, las menores respuestas para variables fisiológicas (Al, PFP, PFB, PSP, PSB), posiblemente un efecto adverso generado por empleo de compostaje (estiércoles avícolas), que presenta altos niveles de calcio, lo que genera antagonismo con cationes, principalmente amonio, magnesio y potasio; derivando una menor disponibilidad y por consiguiente menor aprovechamiento por la planta (Jaramillo, 2001). De otra forma, el fósforo, fundamental en desarrollo y crecimiento de plantas, puede formar compuestos menos solubles y baja disponibilidad en suelo en presencia de altos niveles de calcio (Jaramillo, 2001).

Lo anterior representó que tratamientos orgánicos presentaran menores respuestas fisiológicas frente a las mezclas y la fertilización convencional, se observó en PFP que los tratamientos que presentaron mayor diferencia significativa entre sí fueron Org. min. bajo y Conv. bajo, siendo este último el que mostró una mayor tasa de acumulación de biomasa durante los ciclos, que podría ser por una buena eficiencia fotosintética, provocando un incremento de la biomasa, atribuido a disponibilidad de nitrógeno, ya que en déficit las plantas tienden a disminuir peso, número de hojas y área foliar (Taiz y Zeiger, 2006).

Al mismo tiempo, PFB muestra alta diferencia significativa entre los tratamientos Org. alto y medio, este último mostrando promedio superior al primero, se podría decir que existió mayor transporte de savia elaborada y agua al bulbo, produciendo un incremento en su biomasa, marcando la diferencia con los tratamientos (Taiz y Zeiger, 2006). Los datos obtenidos se hicieron bajo el modelo de un diseño completamente aleatorizado, sin ninguna estratificación.

Sin embargo, la acumulación de fotosintatos no reflejó la condición anterior, como se observa en la variable PSP, en la que no existe ninguna diferencia significativa entre tratamientos, lo cual se explica desde la función de parte aérea, el transporte de nutrientes hacia los órganos de interés, el bulbo de la cebolla, disminuyendo considerablemente peso al eliminarse el contenido de agua (Denisen, 1991).

Por otro lado, el PSP no siempre refleja acumulación de materia seca en el órgano de interés agronómico (bulbo). Al respecto se encontró mayor diferencia entre tratamientos Org. min. bajo y Conv. medio, siendo superior el segundo, indicando que estos bulbos quizás tienen contenido nutricional mucho menor al tratamiento convencional, lo cual puede ser por una mayor absorción de minerales. Por encontrarse estos elementos en mayor disponibilidad, es importante el manejo de la nutrición mineral, práctica que se hace más necesaria debido a sus raíces superficiales y a alta densidad de siembra usada en cebolla (Añez y Tavira, 1986).

Se esperaría que las respuestas en cosecha tuvieran comportamiento similar al presentado durante el ciclo del cultivo, sin embargo se encontró que las fertilizaciones Org. medio y Org. min. bajo presentaron los valores más altos. Los tratamientos Org. medio con un peso promedio por 


\begin{tabular}{|c|c|c|c|c|c|c|c|c|c|c|c|}
\hline Tratamiento & Altura $(\mathrm{cm})$ & $\begin{array}{l}\text { Peso freso } \\
\text { planta }(\mathrm{g})\end{array}$ & $\begin{array}{l}\text { Peso fresco } \\
\text { bulbo }(\mathrm{g})\end{array}$ & $\begin{array}{l}\text { Peso seco } \\
\text { planta }(\mathrm{g})\end{array}$ & $\begin{array}{l}\text { Peso seco } \\
\text { bulbo }(\mathrm{g})\end{array}$ & $\begin{array}{l}\text { Peso fresco } \\
\text { primera }(\mathrm{g})\end{array}$ & $\begin{array}{l}\text { Peso seco } \\
\text { primera }(\mathrm{g})\end{array}$ & $\begin{array}{l}\text { Peso fresco } \\
\text { segunda }(\mathrm{g})\end{array}$ & $\begin{array}{l}\text { Peso seco } \\
\text { segunda }(\mathrm{g})\end{array}$ & $\begin{array}{l}\text { Rendimiento } \\
\text { primera }(\mathrm{kg})\end{array}$ & $\begin{array}{l}\text { Rendimiento } \\
\text { segunda }(\mathrm{kg})\end{array}$ \\
\hline Org. alto & 42,68 a & 30,63 a & $11,90 \mathrm{a}$ & 3,15 a & 1,89 a & 118,68 a & 10,55 a & $47,52 a b$ & $4,53 a b$ & 91,75 a & 30,79 a \\
\hline Org. medio & $45,05 a b$ & $42,76 \mathrm{bc}$ & $14,93 a b$ & 4,10 a & $2,51 a b$ & $158,63 b$ & $17,04 \mathrm{~b}$ & $61,58 \mathrm{c}$ & $6,71 b$ & $168,33 \quad b$ & 26,88 a \\
\hline Org. bajo & $45,67 a b$ & $43,93 \mathrm{~b}$ & $17,81 a b$ & 4,70 a & $3,89 a b$ & 110,47 a & $11,77 a b$ & $46,63 a b$ & $6,15 a b$ & 97,96 a & 31,40 a \\
\hline Org. min. alto & $44,84 a b$ & $33,66 a b$ & $15,58 a b$ & 3,24 a & $2,42 a b$ & 114,58 a & 10,32 a & 42,93 a & $4,61 a b$ & 97,79 a & 36,84 a \\
\hline Org. min. medio & $44,49 a b$ & $37,19 a b c$ & 11,72 a & 3,32 a & 2,03 a & $105,21 a$ & 10,39 a & $45,70 a b$ & 3,56 a & 83,07 a & 32,61 a \\
\hline Org. min. alto & $45,25 a b$ & 31,98 a & $11,81 \mathrm{a}$ & 3,46 a & $2,23 a b$ & $133,88 a b$ & $16,00 a b$ & $53,83 \mathrm{bc}$ & $5,90 a b$ & $136,04 \quad b$ & 47,75 a \\
\hline Conv. alto & $45,38 a b$ & $37,34 a b c$ & $13,16 a b$ & 3,67 a & $2,37 \mathrm{ab}$ & 107,02 a & $13,33 a b$ & $47,35 a b$ & $6,23 a b$ & 88,21 a & 31,23 a \\
\hline Conv. medio & $46,50 \mathrm{~b}$ & $43,19 b c$ & $21,26 b$ & 4,88 a & $4,48 \mathrm{~b}$ & $126,99 \mathrm{ab}$ & $12,41 a b$ & $48,18 a b$ & $5,34 a b$ & $97,38 \quad a$ & 44,96 a \\
\hline Conv. bajo & $44,15 a b$ & $44,55 \mathrm{c}$ & $18,08 a b$ & 4,97 a & $3,52 a b$ & $101,19 a$ & $12,74 a b$ & $44,50 a b$ & 3,77 a & 90,42 a & 27,38 a \\
\hline
\end{tabular}

Promedios con letras distintas en la misma columna indican diferencia significativa según la prueba de Tukey $(P \leq 0,05)$. 
ciclo de 158,63 g y el Org. min. bajo con 133,88 g presentaron un grado de significancia $(P \leq 0,05)$, lo cual encuentra coherencia con lo que reporta García (2001), quien menciona que la incorporación de abonos orgánicos bajo un correcto plan de fertilización y nutrición vegetal tiene respuestas favorables en cuanto a peso de los bulbos.

El comportamiento de PSBP es similar frente al PFBP, para el cual los tratamientos con diferencia significativa $(P \leq 0,05)$ son Org. medio y Org. min. bajo, mostrando respectivamente $17,04 \mathrm{~g} \mathrm{y}$ 15,99 g. En cuanto a esto, Socorro (1994) reporta que el empleo de Azotobacter como parte del plan de fertilización es benéfico para obtener bulbos de primera de mayor peso.

En lo correspondiente a PFBS, los tratamientos que manifestaron mayor peso y mostraron diferencia significativa $(P \leq 0,05)$ fueron Org. medio y Org. min. bajo, alcanzando 61,58 g y 53,83 g, respectivamente, atribuido a un efecto positivo de la adición de compost, ya que según Gardezi et al. (1995) esta estimula la actividad biológica, haciendo que la planta se nutra mejor.

Igualmente, en PSBS se halló diferencia significativa entre los mismos tratamientos, relacionándose concretamente con la variable PFBS, en la que el tratamiento orgánico en dosis media cuenta con mayor calidad, es decir, al tener mayor contenido de materia orgánica es probable que cuente con un contenido nutricional superior a los demás tratamientos (Agamennoi et al., 1998).

Para RP se observó diferencia significativa de los tratamientos Org. medio con rendimiento 22,72 $\mathrm{t}$ ha $\mathrm{a}^{-1}$ y Org. min. bajo con rendimiento $18,35 \mathrm{t}$ $\mathrm{ha}^{-1}$, el cual se encuentra en un rango mayor con respecto al rendimiento nacional de 21,6 t ha $\mathrm{h}^{-1}$ referenciado por Melo et al. (2006). Es de notar que sólo se ha hecho el cálculo para la producción de bulbos de primera.

Para el RS no se presentaron diferencias significativas, como se había mencionado anteriormente entre los tratamientos, indicando homogeneidad en producción de bulbos segunda, lo que representó $23,67 \%$ del total de la producción.

La explicación a los resultados obtenidos en cosecha es atribuible a la frecuencia de aplicación que presentaban los diferentes tratamientos, los orgánicos fueron aplicados al inicio del experimento, algo que generó un efecto contrario en las evaluaciones iniciales de las variables fisiológicas, marcando respuestas negativas en el desarrollo del cultivo, sin embargo, hacia final del ciclo la no aplicación de ningún tipo de fertilizante hizo que no se presentaran antagonismos, evidenciados en la primera parte del ciclo.

Lo anterior puede corroborarse con la respuesta del tratamiento que tuvo la dosis más baja de mezcla orgánico-mineral, en la que sólo se realizaron dos aplicaciones de fertilizante y, contrario a lo encontrado en la fertilización convencional, en la cual al realizarse tres aplicaciones se obtuvieron respuestas inferiores a las esperadas, probablemente a baja disponibilidad de nutrientes generada por exceso de iones en el suelo que incrementa la conductividad eléctrica, generando estrés a la planta (Jaramillo, 2001).

\section{Parámetros edáficos}

En lo referente al suelo, se debe mencionar que las condiciones actuales del suelo distan notablemente de lo esperado en un Humic dystrudept que presentan una reacción fuerte-fuertemente ácida, saturación de aluminio alta y fertilidad moderada a baja, los valores encontrados se aprecian en la tabla 3.

El pH del suelo está cercano a la neutralidad, la acidez intercambiable es mínima, generada por altos niveles de carbono orgánico y la fertilidad de este suelo se puede considerar alta. Lo anterior se presenta por el manejo dado, durante cuatro años, realizando aplicaciones de cales agrícolas de 2,5 $\mathrm{t} \mathrm{ha}^{-1}$ año-1 y de gallinazas en dosis superiores a las $8 \mathrm{t} \mathrm{ha}^{-1}$ por ciclo. Probablemente la 
Tabla 3. Promedio de valores de las propiedades químicas del suelo.

\begin{tabular}{|c|c|c|c|c|c|c|c|c|c|}
\hline \multicolumn{2}{|c|}{$\mathrm{ppm}$} & $\%$ & \multicolumn{9}{|c|}{$\mathrm{cmol}(+) \mathrm{kg}^{-1}$} \\
\hline $\mathrm{pH}$ & $\mathrm{P}$ & $\mathrm{CO}$ & $\mathrm{Ca}$ & $\mathrm{Mg}$ & $\mathrm{K}$ & $\mathrm{Na}$ & $\mathrm{Aci}$ & $\mathrm{CICE}$ & $\mathrm{CIC}$ \\
\hline 6,96 & 946,20 & 3,87 & 39,87 & 2,80 & 3,23 & 0,30 & 0,10 & 46,16 & 32,65 \\
\hline
\end{tabular}

clasificación actual ya no lo coloca en un Humic dystrudept, sino en otra ubicación taxonómica.

Como se observa en la tabla 4, el pH presentó una correlación inversa con las variables fisiológicas (Al, PFP, PSP, PFB, PSB) evaluadas durante el ciclo de cultivo, ocasionando un efecto adverso en el desarrollo vegetativo, mientras que para las variables trabajadas en la cosecha tiene una correlación directamente proporcional con el PFBP, PFBS, PSBP y PSBS, corroborando de esta manera un mejor comportamiento para los tratamientos. En el rango de $\mathrm{pH}$ de 5,5 a 6,5, la solubilidad es la ideal, por debajo de 5,5 el Al puede ser tóxico $y$ en el caso del $\mathrm{P}$ es menos disponible, mientras por encima de 6,5 los elementos menores no son disponibles (Pansu y Gautheyrou, 2003).

Para el segundo ciclo, se cambió la fuente de urea por sulfato de amonio, buscando generar acidez en el suelo y, por ende, mayor disponibilidad de los nutrientes menores para la planta, lo cual se evidenció al finalizar la segunda etapa, en la que se obtuvo un pH entre 6,5 a 6,8, que sigue considerándose neutro.

Esto se puede explicar teniendo en cuenta los procesos químicos que se realizan en el suelo y su influencia sobre la nutrición vegetal, como: disolución, oxidación, hidrolización y reducción (Malagón, 1995).

La disolución de nutrientes puede realizarse en un medio básico o en uno ácido. Al acidificarse la solución del suelo, se disuelven los fosfatos cálcicos, pero tienden a formar los óxidos e hidróxidos de aluminio y de hierro; por otro lado, al dis- minuir la acidez de un suelo, se solubilizan parte de los fosfatos de hierro y aluminio. Por medio de la oxidación, se transforman los sulfuros en sulfatos, y de esta manera, el azufre se hace asimilable para las plantas, elemento de interés en el cultivo de cebolla cabezona (Fassbender, 1980; Zapata, 2006).

El fósforo es el elemento que interviene en todos los procesos fisiológicos, por tanto, tiene alta importancia en la mejora de la calidad de los productos agrícolas (Wild y Jones, 1992).

En el análisis químico se considera que un buen contenido de P se encuentra por encima de las 25 ppm, sin embargo para este caso osciló entre las 800 y 1.100 ppm, mostrando una alta riqueza en este elemento.

Es importante recordar que la disponibilidad de este elemento depende del $\mathrm{pH}$ de la solución del suelo, sin embargo, para el ensayo, al encontrarse entre 6 y 7 , se tiene una condición favorable para la absorción por las plantas (Hanke, 2000).

El P no mostró diferencia significativa $(P>0,05)$ en ninguna de las variables evaluadas, aunque los tratamientos que incluyeron en su fertilización el compost y la roca fosfórica presentaron mayores cantidades de fósforo.

Cabe resaltar que, según Hanke (2000), el abonamiento con materia orgánica tiene generalmente el efecto de un mejor suministro de los fosfatos a los cultivos, ya que la descomposición de ésta, y con ayuda de los microorganismos, mejora la disposición de fósforo para las plantas. 
Tabla 4. Análisis de correlación de las variables de respuestas fisiológicas y algunas propiedades químicas del suelo.

\begin{tabular}{|c|c|c|c|c|c|c|c|c|c|c|c|c|c|c|c|c|c|c|c|c|c|}
\hline & Al & PFP & PFB & PSP & PSB & PFBP & PFBS & PSBP & PSBS & RP & RS & $\mathrm{pH}$ & P & CO & $\mathrm{Ca}$ & $\mathrm{Mg}$ & K & $\mathrm{Na}$ & Acl & CICE & CIC \\
\hline Al & 1 & & & & & & & & & & & & & & & & & & & & \\
\hline PFP & $0,83^{* *}$ & 1 & & & & & & & & & & & & & & & & & & & \\
\hline PFB & $0,52^{* *}$ & $0,66^{* *}$ & 1 & & & & & & & & & & & & & & & & & & \\
\hline PSP & $0,69^{* *}$ & $0,78^{* *}$ & 0,26 & 1 & & & & & & & & & & & & & & & & & \\
\hline PSB & $0,71^{* *}$ & $0,80^{* *}$ & $0,36^{* *}$ & $0,98^{* *}$ & 1 & & & & & & & & & & & & & & & & \\
\hline PFBP & $-0,40^{* *}$ & $-0,45^{* *}$ & $-0,27$ & $-0,50^{* *}$ & $-0,48^{* *}$ & 1 & & & & & & & & & & & & & & & \\
\hline PFBS & $-0,61^{* *}$ & $-0,51^{* *}$ & $-0,22$ & $-0,55^{* *}$ & $-0,53^{* *}$ & $0,61^{* *}$ & 1 & & & & & & & & & & & & & & \\
\hline PSBP & $-0,37^{* *}$ & $-0,38^{* *}$ & $-0,27^{*}$ & $-0,43^{* *}$ & $-0,43^{* *}$ & $0,68^{* *}$ & $0,61^{* *}$ & 1 & & & & & & & & & & & & & \\
\hline PSBS & $-0,44^{* *}$ & $-0,42^{* *}$ & $-0,20$ & $-0,44^{* *}$ & $-0,43^{* *}$ & $0,46^{* *}$ & $0,67^{* *}$ & $0,63^{* *}$ & 1 & & & & & & & & & & & & \\
\hline RP & $0,31^{*}$ & $0,27^{*}$ & 0,19 & 0,24 & 0,21 & | 0,27 & 0,13 & $0,29^{*}$ & 0,09 & 1 & & & & & & & & & & & \\
\hline RS & $-0,21$ & $-0,26$ & $-0,21$ & $-0,20$ & $-0,21$ & 0,12 & 0,15 & $0,33^{*}$ & 0,17 & $-0,01$ & 1 & & & & & & & & & & \\
\hline $\mathrm{pH}$ & $-0,55^{* *}$ & $-0,51^{* *}$ & $-0,58^{* *}$ & $-0,29^{*}$ & $-0,31^{*}$ & $0,42^{* *}$ & $0,51^{* *}$ & $0,40^{* *}$ & $0,48^{* *}$ & $-0,19$ & 0,06 & 1 & & & & & & & & & \\
\hline $\mathrm{P}$ & 0,14 & 0,11 & 0,03 & 0,24 & 0,22 & $-0,30^{*}$ & $-0,32^{*}$ & $-0,30^{*}$ & $-0,31^{*}$ & 0,1 & $-0,24$ & 0,02 & 1 & & & & & & & & \\
\hline co & 0,06 & 0,07 & $-0,12$ & 0,09 & 0,07 & $-0,16$ & $-0,11$ & 0,02 & $-0,10$ & $-0,02$ & 0,07 & $-0,07$ & $0,32^{*}$ & 1 & & & & & & & \\
\hline $\mathrm{Ca}$ & $0,67^{* *}$ & $0,62^{* *}$ & $0,47^{* *}$ & $0,43^{* *}$ & $0,46^{* *}$ & $-0,33^{*}$ & $-0,26$ & $-0,28^{*}$ & $-0,17$ & 0,23 & $-0,23$ & $-0,36^{* *}$ & $0,29^{*}$ & 0,25 & 1 & & & & & & \\
\hline $\mathrm{Mg}$ & 0,20 & 0,06 & $-0,17$ & 0,24 & 0,25 & $-0,11$ & $-0,28^{*}$ & $-0,03$ & $-0,27^{*}$ & 0,09 & $-0,01$ & 0,03 & $0,42^{* *}$ & 0,23 & 0,11 & 1 & & & & & \\
\hline $\mathrm{K}$ & $0,68^{* *}$ & $0,58^{* *}$ & $0,54^{* *}$ & 0,22 & 0,24 & $-0,21$ & $-0,43^{* *}$ & $-0,19$ & $-0,32^{*}$ & $0,30^{*}$ & $-0,20$ & $-0,64^{* *}$ & 0,15 & 0,12 & $0,59^{* *}$ & 0,11 & 1 & & & & \\
\hline $\mathrm{Na}$ & 0,25 & $0,38^{* *}$ & $0,41^{* *}$ & 0,21 & $0,27^{*}$ & $-0,12$ & $-0,29^{*}$ & $-0,24$ & $-0,22$ & 0,02 & $-0,09$ & $-0,17$ & 0,07 & $-0,19$ & 0,24 & $-0,03$ & 0,15 & 1 & & & \\
\hline Acl & $-0,19$ & $-0,10$ & $-0,01$ & $-0,09$ & $-0,07$ & 0,10 & 0,08 & 0,22 & $-0,02$ & 0,01 & $0,29^{*}$ & $-0,08$ & 0,06 & $-0,06$ & $-0,31^{*}$ & 0,04 & $-0,10$ & $-0,16$ & 1 & & \\
\hline CICE & $0,70^{* *}$ & $0,64^{* *}$ & $0,49^{* *}$ & $0,43^{* *}$ & $0,46^{* *}$ & $-0,33^{*}$ & $-0,31^{*}$ & $-0,29^{*}$ & $-0,21$ & 0,26 & $-0,23$ & $-0,40^{* *}$ & $0,31^{*}$ & 0,25 & $0,99^{* *}$ & 0,16 & $0,66^{* *}$ & 0,26 & $-0,29^{*}$ & 1 & \\
\hline CIC & $-0,42^{* *}$ & $-0,37^{* *}$ & $-0,24$ & $-0,35^{* *}$ & $-0,37^{* *}$ & 0,17 & $0,35^{* *}$ & 0,19 & 0,26 & $-0,24$ & 0,06 & $0,31^{*}$ & 0,19 & 0,12 & $-0,09$ & $-0,01$ & $-0,30^{*}$ & $-0,26$ & $0,40^{* *}$ & $-0,12$ & 1 \\
\hline
\end{tabular}




\begin{tabular}{|l|c|c|c|c|c|}
\hline \multicolumn{6}{|c|}{ Tabla 5. Efecto de la fertilización sobre algunos grupos microbiológicos (en ufc/g de suelo seco a la dilución de 10). } \\
\hline \multicolumn{1}{|c|}{ Tratamiento } & Fijadoras de N & Solubilizadores de P & Hongos & Actinomicetos & Bacterias \\
\hline Org. alto & $87,25 \mathrm{abc}$ & $57,25 \mathrm{a}$ & $75,00 \mathrm{a}$ & $35,16 \mathrm{a}$ & $99,25 \mathrm{a}$ \\
\hline Org. medio & $95,08 \mathrm{abc}$ & $47,83 \mathrm{a}$ & $55,91 \mathrm{a}$ & $57,58 \mathrm{a}$ & $80,75 \mathrm{a}$ \\
\hline Org. bajo & $105,83 \mathrm{c}$ & $53,66 \mathrm{a}$ & $93,25 \mathrm{a}$ & $34,08 \mathrm{a}$ & $91,83 \mathrm{a}$ \\
\hline Org. min. alto & $90,91 \mathrm{abc}$ & $47,50 \mathrm{a}$ & $88,66 \mathrm{a}$ & $39,41 \mathrm{a}$ & $90,41 \mathrm{a}$ \\
\hline Org. min. medio & $66,83 \mathrm{abc}$ & $53,91 \mathrm{a}$ & $58,08 \mathrm{a}$ & $45,50 \mathrm{a}$ & $103,83 \mathrm{a}$ \\
\hline Org. min. bajo & $63,91 \mathrm{ab}$ & $56,91 \mathrm{a}$ & $49,83 \mathrm{a}$ & $43,25 \mathrm{a}$ & $73,75 \mathrm{a}$ \\
\hline Conv. alto & $84,91 \mathrm{abc}$ & $47,75 \mathrm{a}$ & $93,58 \mathrm{a}$ & $19,66 \mathrm{a}$ & $100,08 \mathrm{a}$ \\
\hline Conv. medio & $99,41 \mathrm{~b}$ & $49,00 \mathrm{a}$ & $84,16 \mathrm{a}$ & $51,25 \mathrm{a}$ & $98,50 \mathrm{a}$ \\
\hline Conv. bajo & $55,75 \mathrm{a}$ & $48,00 \mathrm{a}$ & $109,91 \mathrm{a}$ & $37,08 \mathrm{a}$ & $84,83 \mathrm{a}$ \\
\hline
\end{tabular}

Promedios con letras distintas en la misma columna indican diferencia significativa según la prueba de Tukey $(P \leq 0,05)$.

Otro factor importante que atañe a este elemento es el tipo de arcillas del suelo. Para el ensayo se encontró que, al ser de un origen ándico, es posible la presencia de alófanas, las cuales pueden llegar a fijar este elemento hasta un $85 \%$, no obstante al poseer tan altas cantidades aún se tendría una buena cantidad disponible a las plantas.

Revisando la correlación, se encuentra este elemento vinculado de manera significativa e inversa con la producción de cebolla de segunda, lo que se le podría atribuir a la ley de los rendimientos decrecientes, en la que se indica que, a medida que aumenta el insumo (fertilizante), se llega a un punto en que la producción disminuye (Fremont, 1961; Zapata, 2006).

La necesidad del cultivo en calcio ( $\mathrm{Ca}$ ) es baja, hasta en suelos ácidos no se restringe la disponibilidad de iones de Ca para abastecer las exigencias de la cebolla, además varios fertilizantes lo contienen como componente secundario.

Al lote donde se trabajó, por varios años se le ha incorporado cantidades altas de cal. Sin haber realizado algún análisis de suelo, se encontró un porcentaje de saturación mayor al $80 \%$. Se encontró correlación directa entre los niveles de calcio en el suelo con la producción. Por otra parte, altos contenidos de este elemento inhiben la absorción de $\mathrm{Fe}, \mathrm{B}, \mathrm{Zn}$, Cu y Mg, porque estos elementos pierden su solubilidad con un $\mathrm{pH}$ mayor de seis (Zapata, 2006).

El contenido de magnesio $(\mathrm{Mg})$ se encontró por debajo de los niveles ideales, localizándose en un rango de 6 a $8 \%$ de saturación, lo cual se debe a la interacción con el calcio, que, como ya se mencionó, inhibe otros elementos, por su alta presencia en el suelo.

Se encontró además correlacionado de manera altamente significativa y positiva con la altura de la planta, lo cual se manifiesta, posiblemente, porque el Mg forma parte de la molécula esencial de las plantas (clorofila) y se fomenta el crecimiento y desarrollo vegetativo de éstas (Wild y Jones, 1992).

El potasio $(\mathrm{K})$ tiene una relación altamente significativa y positiva con las siguientes variables: altura, peso fresco y seco de planta y bulbo, de manera un poco menos significativa con la producción de bulbo de primera; ya que este elemento se encontró por encima del óptimo de saturación en el suelo, lo que reporta incluso protección contra ataque de hongos, porque este elemento forma fibras más fuertes, haciendo a las plantas más resistente contra este tipo de ataques, además crea resistencia contra la sequia, ya que disminuye la traspiración (Hanke, 2000). 
Dogliotti y Galvan (2008) determinan que para el uso entre cloruros o los sulfatos se debe tener presente el cultivo, ya que plantas como la papa, el tabaco, frutales y hortalizas prefieren los sulfatos, mientras que plantas halófitas, como la remolacha, la caña de azúcar y forrajes, prefieren los cloruros.

El sodio $(\mathrm{Na})$ no se considera un elemento esencial, pero puede reemplazar algunas funciones del K. Se observa una alta correlación de Pearson entre el el peso fresco de la planta y el bulbo.

\section{Parámetros microbiológicos}

Las bacterias se encontraron en abundancia en todos los tratamientos y no mostraron diferencias entre los tratamientos, ni entre los ciclos.

En cuanto a la correlación de Pearson, se hallaron relacionadas con un nivel de significancia de 0,05 de forma directa con las siguientes variables: altura, peso fresco de planta y bulbo, además de manera inversa se correlacionaron con el $\mathrm{pH}$. Esto último se puede dar debido a que las bacterias prefieren $\mathrm{pH}$ cercanos a la neutralidad; en el experimento, el $\mathrm{pH}$, a pesar de encontrarse en un rango de neutralidad, pasó de 7,2 a 6,5, por lo que se diría que son susceptibles a estas variaciones, ya que se incrementaron en el segundo ciclo, pasando en promedio de 83 ufc a 100 ufc.

En lo referente a los actinomicetos, es importante reconocer que los tratamientos Org. medio y Org. min. bajo nuevamente mostraron mayor cantidad de estos microorganismos, mostrando su intrínseca relación con el desarrollo y productividad de las plantas.

Con este grupo de microorganismos se encontró una correlación inversa $(P \leq 0,05)$ con el carbono orgánico y el magnesio, lo que puede darse porque algunos actinomicetos contribuyen a la precipitación de $\mathrm{CaCO}_{3}$ en el suelo, alterando de esta manera el pH (Damian, 1992).
Durante el estudio, los hongos no mostraron diferencia significativa para el número de sus poblaciones, sin embargo los tratamientos que evidenciaron una mayor riqueza en estos microorganismos fueron los convencionales en sus tres dosis, estando inclusive por encima de las bacterias en el tratamiento Conv. bajo (hongos 33\% y bacterias $25 \%$ ).

Los solubilizadores de fósforo presentaron mejores respuestas en cuanto a los tratamientos con mejor rendimiento, fueron aquellos que coinciden con la mejor presencia de este tipo de microorganismos.

El porcentaje promedio que se manejó de estas poblaciones en los diferentes tratamientos fue de $15 \%$, el cual es considerado bueno, teniendo en cuenta la intensa actividad microbiológica que aportan al suelo.

En cuanto a las fijadoras de nitrógeno, la correlación de Pearson se determinó relacionada con todas las variables fisiológicas en forma altamente significativa y directa, indicando que, a mayor proporción de ufc de estos microorganismos en el suelo, la planta presenta una mejor nutrición que conlleva a un óptimo desarrollo vegetativo y reproductivo.

\section{CONCLUSIONES}

El suelo del estudio demostró mediante los análisis químicos que el manejo convencional de éstos ha generado una modificación de las condiciones naturales, elevando las cantidades de los elementos por encima de los niveles críticos, por lo cual posiblemente no se requieran aplicaciones de fertilizantes de forma continua.

Los suelos enriquecidos por aplicaciones de materia orgánica y enmiendas con cales generan niveles de elementos por encima de niveles críticos, en los que la respuesta en la producción se atribuye más a una mejora en la condición química del suelo que a un aporte de nutrientes a través de la fertilización. 
El análisis de suelo forma parte del manejo de la fertilización y debe considerarse como una buena herramienta para la toma de decisiones.

Es posible que el aumento de los microorganismos se haya debido a condiciones climáticas de la zona en el tiempo de evaluación y no a los tratamientos empleados, ya que se observaron poblaciones de éstos inclusive en tratamientos como el convencional, por lo que se cree que es más un efecto ambiental que del experimento.

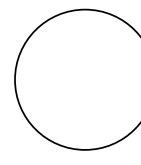

Abril, A. 2002. La microbiología del suelo: su relación con la agricultura sustentable. pp. 129-150. En: Sarandon, S.J. (ed.). Agroecología. El camino hacía una agricultura sustentable. Ediciones Científicas Americanas, La Plata, Argentina.

Agamennoi, R.; J. Rivas; R. Delhey y A. Azpilicueta. 1998. Abonos verdes de verano en la producción de cebolla. Resúmenes XXI Congreso Argentino de Horticultura. San Pedro, Buenos Aires.

Añez, B. y E. Tavira. 1986. Aplicación de N, P y K a diferentes poblaciones de plantas de cebolla. Turrialba 36(2), 163-169.

Borrero, S. 2000. Estudio general de suelos y zonificación de tierras. IGAC, Bogotá.

Burbano, H. 1989 El suelo: una visión sobre sus componentes biorgánicos. Universidad de Nariño, Pasto, Colombia.

Damian, C.A. 1992. Actinomicetos y su efecto antagónico sobre Phytophtora cinnamomi Rands (tristeza del aguacate) bajo la incorporación de dos mejoradores orgánicos del suelo y un fungicida. Tesis. Departamento de Parasitología Agrícola, Universidad Autónoma de Chapingo, México.

Denisen, E.L. 1991. Fundamentos de la horticultura. 2a. ed. Editorial Limusa, México.

Dogliotti, S. y G. Galvan. 2008. Bases fisiológicas del crecimiento y desarrollo de cultivo de cebolla (Allium
La microbiota edáfica es un factor bastante relevante a considerar cuando se planea el establecimiento del cultivo. Ya que el establecimiento de una flora microbiana permite una mayor asimilación de los nutrientes por las plantas y, por tanto, la cantidad de fertilizantes a utilizar puede llegar a reducirse.

Es necesario realizar más investigación sobre los grupos de microorganismos, ya que por su capacidad de producir sustancias antagonistas y benéficas (Gutiérrez, 2000) se podría explotar en mayor proporción su potencial en agricultura y en la industria.

\section{REFERENCIAS BIBLIOGRÁFICAS}

cepa L.). p. 19. En: Facultad de Agronomía, Universidad de la Republica, Uruguay, http://www.fagro. edu.uy/ cultivos/hortalizas/Repartido_Fisiologia_Cebolla.pdf; consulta: marzo de 2009.

Fassbender, H.W. 1980. Quimica de los suelos. IICA, San José, Costa Rica.

Ferrera-Cerrato, R. y A. Alarcon. 2007. Microbiologia agricola. Hongos, bacterias, micro y macrofauna, control biológico y planta-microorganismo. Trillas, México.

Fremont, P. 1961. Economía agrícola. Aguilar, Madrid.

García, J. 1963. Fertilización agrícola. Ediciones Agrociencia, Zaragoza, España.

García, M. 2001. Evaluación de la respuesta de una sucesión de cultivos hortícolas a diferentes abonos orgánico. Universidad de la Republica, Montevideo.

Gardezi, A.K. 1995. Endomycorrhiza, rock phosphate, and organic matter effects on growth of Erythrina americana. Nitrogen Fixing Tree Res. 13, 48-50.

Gutiérrez, L.M.T. 2000. Potencial herbicida de metabolitos aislados de especies selectas de actinomicetos. Actinomyces MIV2B (31) como una fuente novedosa del antibiótico maduramicina. Tesis de Doctorado. UNAM, México.

Hanke, F. 2000. La nutrición de la planta y su problemática en la agricultura. Edición Talleres Gráficos Fundación Universitaria Juan de Castellanos, Tunja, Colombia. 
Instituto Colombiano Agropecuario. 1989. Análisis de suelos, plantas y aguas para riego. ICA, Bogotá.

Jaramillo, D. 2001. Introducción a la ciencia del suelo. Escuela de Geociencias, Universidad Nacional de Colombia, Medellín, Colombia.

Malagón, D.; C. Pulido; R. Llinas y C. Chamorro. 1995. Suelos de Colombia, origen, evolución, clasificación, distribución y uso. Canal Ramírez Antares, Bogotá.

Melo, L.I.; M.M. Melo y L.F. Rodríguez. 2006. Competitividad del sistema agroalimentario de la cebolla de bulbo con enfoque de cadena productiva en el distrito de riego del alto Chicamocha (Boyacá). Agron. Colomb. 24(2), 378-389.

Pansu, M. y J. Gautheyrou. 2003. Handbook of soil analysis. Mineralogical, organic and inorganic methods. Springer-Verlag, Berlin.
Scharrer, K.1960. Química agrícola. Editorial Hispano Americana, México.

Socorro, M.; N. Bouza; O. Morales; P. Meneses; R. Cabello y B.Dibut. 1994. Empleo del Azotobacter chroococcum en la fertilización nitrogenada del arroz. p. 113. En: Resúmenes XVII Reunión Latinoamericana de Rhizobiología. La Habana.

Wild, A. y L.H.P. Jones. 1992. Nutrición mineral de las plantas cultivadas. pp. 73-119. En: Wild. A. (ed.). Condiciones del suelo y desarrollo de las plantas según Russel. Ediciones Mundi-Prensa, Madrid.

Taiz, L. y E. Zeiger. 2006. Plant physiology. $4^{\text {th }}$ ed. Sinauer Associates Publ., Massachussetts, MA.

Zapata, H. 2006. Química de los procesos. Pedogenéticos. Universidad Nacional de Colombia, Medellín, Colombia. 